\title{
Human Body Modeling for Riding Comfort Simulation
}

\author{
Hyung Yun $\mathrm{Choi}^{1}$, Kyung Min Kim ${ }^{1}$, Jiwon $\mathrm{Han}^{1}$, Sungjin $\mathrm{Sah}^{1}$, Seok-Hwan Kim ${ }^{2}$, \\ Su-Hwan Hwang ${ }^{2}$, Kwang No Lee ${ }^{2}$, Jong-Kweon Pyun ${ }^{2}$, Nicole Montmayeur ${ }^{3}$, \\ Christian Marca ${ }^{3}$, Eberhard $\mathrm{Haug}^{3}$, and Inhyeok Lee ${ }^{3}$ \\ ${ }^{1}$ Hongik University, ME Dept. Seoul, Korea \\ hychoi@hongik.ac.kr \\ ${ }^{2}$ Hyundai-Kia Motor Company, Korea \\ ${ }^{3}$ ESI Group, France
}

\begin{abstract}
In order to assess the seating and riding comfort design of a vehicle seat in an objective manner, finite element occupant models with anatomically precise features have been developed. The human body models are incorporated into Pam-Comfort, a tool for automotive seat design and analysis, as parts of occupant surrogates that also include HPM I and II. In this paper, the detailed process of FE human body modeling including an effort on the implementation of new anthropometry will be introduced. The validation of some features of human body models in seating and riding comfort simulation against human experiments will be also presented.
\end{abstract}

Keywords: Seating and riding comfort, Pam-Comfort, Finite element human body model, Anthropometry.

\section{Introduction}

The design of a comfort seat which earns a good market evaluation needs to be based on the recent anthropometric information of the population so that it can minimize the discomfort feeling possibly caused by failing to fit the diverse sizes and shapes of current occupants. The human body models in PAM-Comfort, a simulation tool for virtual seat design and testing, are in the process to be equipped with new anthropometry obtained from SizeUSA, a nationwide survey in the US [1]. There are three targets in size, two from the edges in "normal" distribution curve that are the $5^{\text {th }}$ percentile female and the $95^{\text {th }}$ percentile male and one from the center which is the $50^{\text {th }}$ percentile male. Using the extreme size such as $5^{\text {th }}$ percentile female and the $95^{\text {th }}$ percentile male in a design process is to minimize the discomfort, e.g. from too wide or too deep a seat pan, while the medium size is testing maximum comfort features. Only caucasian between 36 and 45 years old were retained for the further considerations from the SizeUSA database for narrowing down the analysis to the majority in the USA.

The acquisition of the skin geometry for each model was done by taking the threedimensional whole body laser scans of volunteers in driving position (the $5^{\text {th }}$ percentile female model is not completed at present). For the recruitment of volunteers with representative body size for each target group, a statistical factor 
analysis with the SizeUSA database was performed to determine several primary dimensions. The primary dimensions are classified into three orthogonal factors which can also provide overall shape information of each target group.

In order to build the finite element models with anatomically detailed skeletal structure, medical images such as X-ray pictures and ultrasonic scan images were taken from volunteers to gauge the flesh margins. Body pressure distribution on urethane seat foam was also measured for the model validation.

\section{Anthropometry}

\subsection{SizeUSA Survey Data}

The overall size and shape of the people change with time. They are taller and larger than 30 years ago. The current human body model [2-4], a $50^{\text {th }}$ percentile adult male in Pam-Comfort is based on the anthropometry of a motor vehicle occupant developed in the early 80's [5]. Therefore, a recent US national size survey, SizeUSA [1] has been adopted in order to update the human body model in Pam-Comfort. In the SizeUSA survey performed from 2000 to 2003, the anthropometries of 10,800 subjects (18-65 years old) were directly obtained by taking 3D laser scans of individuals in a normal standing position. Body measurement extraction software was then used to produce a list of body measurements such as length, width, and girth of body segments from the $3 \mathrm{D}$ body data.

There are 3 targets in size, two from the edges in the "normal" distribution curve which are the $5^{\text {th }}$ percentile female and the $95^{\text {th }}$ percentile male and one from the center which is the $50^{\text {th }}$ percentile male. Caucasian between 36 and 45 years old were selected from the SizeUSA database for narrowing down the analysis to the majority in the country. Among 10,800 subjects in total, 687 and 354 were used for female and male objects, respectively. Fig. 1 shows whole body scans for a typical small $\left(5^{\text {th }}\right.$ percentile) female, medium size $\left(50^{\text {th }}\right.$ percentile) male and large size $\left(95^{\text {th }}\right.$ percentile $)$ male from the SizeUSA survey. The corresponding height and weight of each size are also listed in Fig. 1. From the statistical factor analysis, 6 dimensions, i.e., height, weight, hip height, hip girth, bust, and back waist length were selected as primary dimensions among 18 segmental measurements. The 6 primary dimensions are classified into 3 factors which exhibit high orthogonality to each other and thus can provide overall shape information of the population. The results of factor analysis for both male and female are listed in Table 1.

\subsection{Volunteer Recruitment and Selection}

The specific ranges for three target sizes of each primary dimension were assigned as the selection criteria of volunteers for data collection of body shapes. There was a two-stage recruitment process of volunteers. Since people are well aware of their height and weight but not the other four dimensions, volunteers were invited on the condition of specified range of those two dimensions. The ranges of height and weight were set to $\pm \sigma / 4$ (one quarter of standard deviations for small female and 

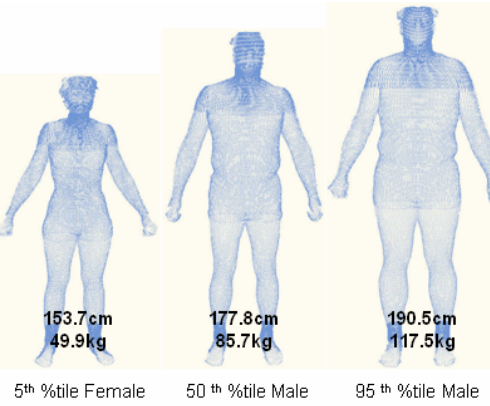

Fig. 1. Typical whole body scans of three target sizes and their standard sizes

Table 1. Results of factor analysis using SizeUSA

\begin{tabular}{|c|c|c|c|c|c|c|}
\hline & \multicolumn{3}{|c|}{ Male } & \multicolumn{3}{c|}{ Female } \\
\cline { 2 - 7 } & F1 & F2 & F3 & F1 & F2 & F3 \\
\hline Height & 0.915 & -0.265 & 0.191 & 0.884 & -0.354 & 0.212 \\
\hline Back Neck Height & 0.951 & -0.234 & 0.147 & 0.925 & -0.314 & 0.159 \\
\hline Long Shoulder Height & 0.937 & -0.250 & 0.132 & 0.915 & -0.322 & 0.130 \\
\hline Hip Height & 0.925 & -0.256 & -0.094 & 0.920 & -0.236 & -0.147 \\
\hline Top Hip Height & 0.924 & -0.298 & -0.212 & 0.944 & -0.231 & -0.202 \\
\hline Upper Hip Height & 0.907 & -0.312 & -0.263 & 0.941 & -0.225 & -0.225 \\
\hline Waist Height & 0.882 & -0.321 & -0.308 & 0.931 & -0.218 & -0.244 \\
\hline Arm Length Straight & 0.847 & -0.266 & 0.095 & 0.791 & -0.239 & 0.052 \\
\hline Knee Height & 0.828 & -0.423 & 0.013 & 0.777 & -0.403 & -0.079 \\
\hline Crotch Height & 0.772 & -0.546 & -0.007 & 0.762 & -0.597 & -0.066 \\
\hline Hip Girth & 0.532 & 0.784 & -0.123 & 0.539 & 0.815 & -0.007 \\
\hline Bust & 0.474 & 0.777 & -0.038 & 0.443 & 0.793 & -0.029 \\
\hline Thigh_Max & 0.540 & 0.759 & -0.037 & 0.535 & 0.786 & 0.033 \\
\hline Thigh_Mid1 & 0.479 & 0.751 & -0.016 & 0.479 & 0.790 & 0.032 \\
\hline Weight & 0.662 & 0.717 & 0.019 & 0.662 & 0.717 & 0.019 \\
\hline Back Shoulder Width & 0.482 & 0.691 & 0.036 & 0.458 & 0.684 & 0.065 \\
\hline Hip Width & 0.574 & 0.666 & -0.077 & 0.587 & 0.690 & 0.034 \\
\hline Back Waist Length & 0.447 & 0.064 & 0.883 & 0.320 & -0.219 & 0.913 \\
\hline
\end{tabular}

medium male) and $\pm \sigma / 2$ (half of standard deviations for large male) from average values and used in local advertisement to recruit Americans staying in Korea. Table 2 lists the ranges of height and weight used in the volunteer recruitment. The visiting volunteers were further screened to be selected with the range of four other primary dimensions shown in Table 3. The upper and lower limits of four primary dimensions represent the respective maximum and minimum dimensions obtained from the data of the subjects in SizeUSA those who satisfied both height and weight. Two Caucasian volunteers of 44 and 43 years old were finally selected for $50^{\text {th }}$ and $95^{\text {th }}$ percentile objects, respectively. Both of them meet the required ranges for the segmental dimensions in Table 3. 
Table 2. Height and weight ranges for volunteer recruitments

\begin{tabular}{|c|c|c|c|c|c|}
\hline & \multirow{2}{*}{$\begin{array}{c}\text { No. of } \\
\text { subjects* }\end{array}$} & \multicolumn{2}{|c|}{ Height $(\mathrm{cm})$} & \multicolumn{2}{c|}{ Weight $(\mathrm{kg})$} \\
\cline { 3 - 5 } & 19 & Range & S.D. $(\sigma)$ & Range & S.D. . . \\
\hline Small female** & $152.1-155.3$ & 6.4 & $45.5-54.3$ & 17.4 \\
\hline Medium male** & 17 & $175.9-179.9$ & \multirow{2}{*}{7.7} & $81.5-89.9$ & \multirow{2}{*}{16.8} \\
\hline Large male*** & 6 & $186.7-194.3$ & & $109.8-125.9$ & \\
\hline
\end{tabular}

* Number of subjects who satisfied both height and weight ranges.

$* *$ avg. $-\sigma / 4 \sim$ avg. $+\sigma / 4, * * *$ avg. $-\sigma / 2 \sim$ avg. $+\sigma / 2$.

$* * * \sigma / 2$ was used since no subjects were found with $\sigma / 4$ among 354 male subjects in SizeUSA.

Table 3. Ranges for other primary dimensions for volunteer selection

\begin{tabular}{|c|c|c|c|}
\hline Dimension $\backslash$ Size & $5^{\text {th }} \%$ tile female & $50^{\text {th }} \%$ tile male & $95^{\text {th }} \%$ tile male \\
\hline Hip Height & $73.2-79.7$ & $87.0-92.5$ & $93.9-103.5$ \\
\hline Back Waist Length & $36.3-44.0$ & $47.6-54.8$ & $44.8-58.3$ \\
\hline Bust Girth & $83.1-99.6$ & $105.2-117.0$ & $111.9-128.5$ \\
\hline Hip Girth & $87.4-103.0$ & $100.0-108.0$ & $110.4-125.7$ \\
\hline
\end{tabular}

\section{Three-Dimensional Scanning and CAD Modeling}

The external skin and internal skeletal geometries of two volunteers were obtained by using three-dimensional scanning devices. Merging these external and internal images of each volunteer, geometrically precise CAD models were constructed.

\subsection{Three-Dimensional Laser Scanning}

The external geometry of selected volunteers in driving posture was measured by a whole body 3D laser scanner (Model: Cyberware WB4). Undeformed shape of buttock and thigh was separately scanned from the volunteers propping on elbows while maintaining the knee angles the same as in driving posture. The synthesis between patches of undeformed parts and the whole body scan image was carried out by using a 3D scan modeling software, RapidForm [www.rapidform.com]. Fig. 2 shows some pictures of volunteer scanning process.
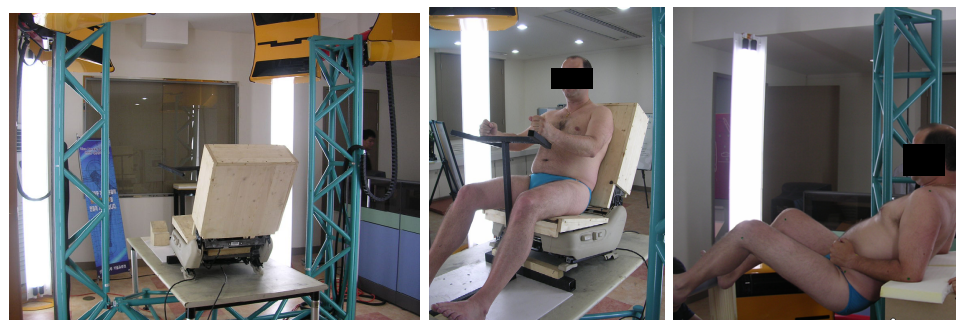

Fig. 2. Whole body 3D scanning of a volunteer in a driving posture 


\subsection{CAD Modeling}

The scanned surface model was reflected along the sagittal plane to make it symmetric. The image with two right sides showed better match ratio with the original scanned image than the one with two left sides. Smoothing the local details, the reflected image was simplified and converted into a surface mesh model. Fig. 3 shows both the original scanned and the reflected images of the 50\%tile percentile male volunteer. The surface mesh model is also depicted in the Fig. 3.

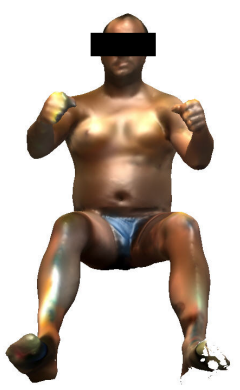

Original image

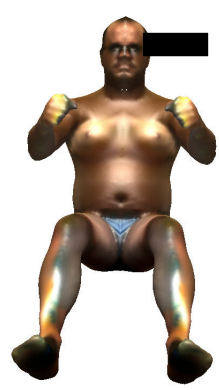

Reflected image
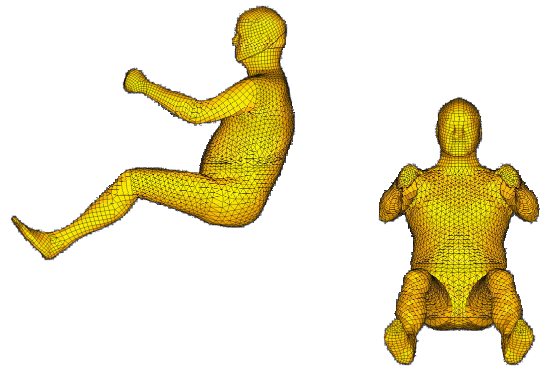

Surface mesh model

Fig. 3. Scanned images and surface mesh model of $50^{\text {th }}$ percentile male volunteer

The precise location of the skeleton to the skin surface in the model is important for the accurate prediction of sitting pressure distributions in the finite element analysis. In order to gauge the flesh margin, an ultrasonic scanning as shown in Fig. 4 was employed. Ultrasonic scanning was performed over the trunk, hip, and thigh regions of the volunteers in a driving position. Both compressed and uncompressed bone depths were measured in particular for the ischial tuberosity where the peak sitting pressure occurs and thus could be an adequate point for a validation of the finite element model and its prediction. X-ray radiography was also taken but used as supportive information. CT scan was not an effective measure since it was limited to scan the body in a supine position.
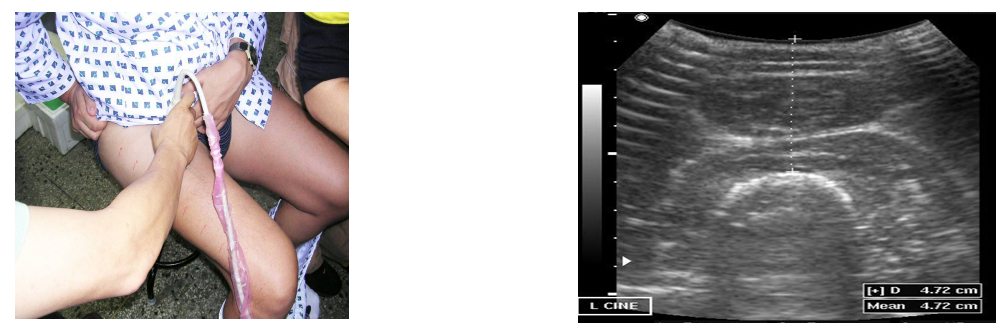

Fig. 4. Ultrasonic scanning for gauging a flesh margin

The locations of joints relative to the surface landmarks were calculated using simple linear scaling relationships [6-8]. Fig. 5 shows schematic drawings of the 


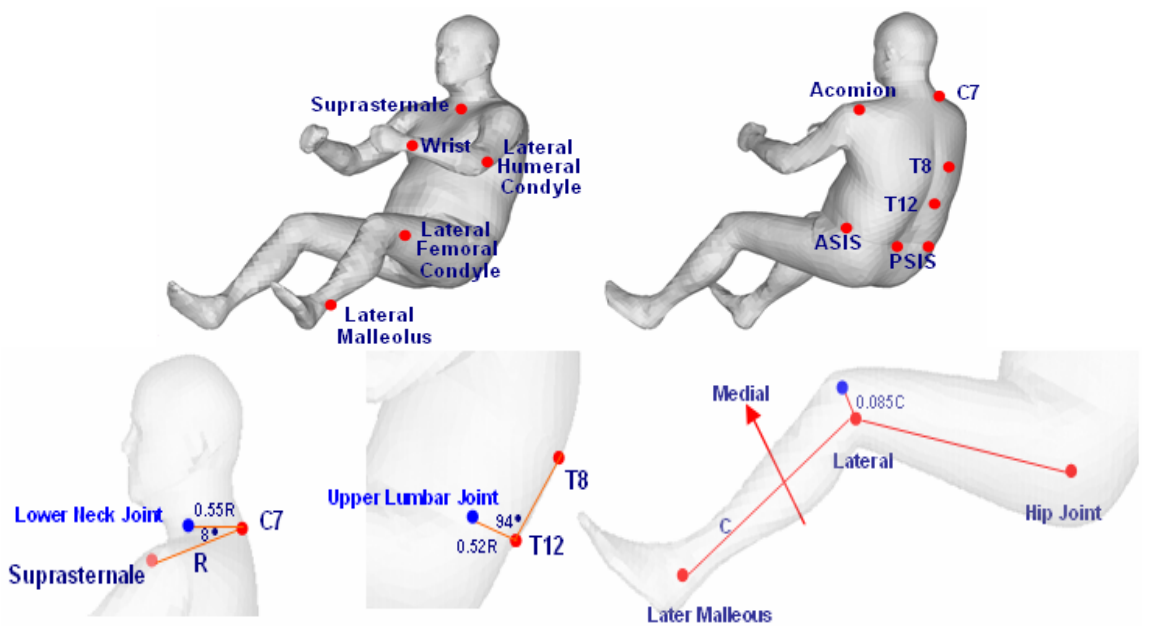

Fig. 5. Schematic drawing of the procedure for calculating various joint locations from surface markers

Table 4. Landmarks for the calculation of joint locations

\begin{tabular}{|c|c|}
\hline Joints & Landmarks \\
\hline $\begin{array}{c}\text { Upper Neck } \\
\text { (atlanto-occipital) }\end{array}$ & Infraorbitale., Tragion \\
\hline Lower Neck (C7/T1) & C7, Suprasternale \\
\hline Upper Lumbar (T12/L1) & T8, T12, C7, Suprasternale \\
\hline Lower Lumbar (L5/S1) & ASIS, PS, PSIS \\
\hline Hip & ASIS, PS, PSIS \\
\hline Shoulder & Acromion, C7, Suprasternale \\
\hline Knee & Lateral Femoral Condyle, Hip joint, lateral malleolus \\
\hline Elbow & Lateral humeral Condyle, shoulder joint, wrist landmark \\
\hline Wrist & Wrist \\
\hline Ankle & Lateral malleolus, lateral femoral condyle, hip joint \\
\hline
\end{tabular}
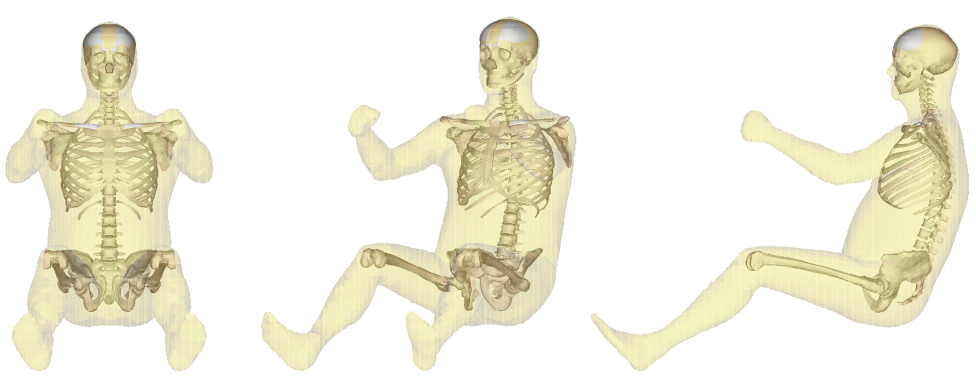

Fig. 6. Skin with skeleton for $50^{\text {th }}$ percentile male model 
procedure for calculating various joint locations from surface markers. Table 4 lists the joint locations of the model associated with landmarks used in the calculation.

A generic skeletal structure, which was used for H-model [9] was incorporated into the skin model by scaling it to match the flesh margins as well as the joint locations computed as in Fig 5. The skin model with skeleton inside for the $50^{\text {th }}$ percentile male volunteer is shown in Fig. 6.

\section{Construction of Comfortable Driving Posture}

A driving posture is to be determined according to the design of the interior package layout and the operating pattern of a driver. A certain level of fatigue might be produced as the consequence of an uncomfortable driving posture. Therefore, both the package layout of vehicle interior and the anthropometry of a driver need to be taken into account simultaneously to set up the standard seating posture in the design process. Reed [10] proposed the Cascade Prediction Model (CPM), which consists of linear regression equations and inverse kinematics to build a comfortable driving posture. The linear regression equation based on the major factors of package layout and anthropometric data provides eye and hip locations of a given driver. Segmental angles in a comfortable posture can then be optimally obtained from an inverse kinematics computation. The CPM was constructed from the volunteer tests with 68 subjects taking 916 numbers of comfortable voluntary postures. Seat height, steering wheel position, and cushion angles were selected as independent variables affecting the driving posture. In order to make the model posture changeable, the skin model was segmented into 15 parts, i.e. head, neck, upper torso, abdomen, hip, two upper arms, two forearms (including hands), two upper legs, and two lower legs, and two feet, which are articulated at the joints. This kinematic linkage model was visualized by adding a number of stick elements between each joint location as shown in Fig. 7.

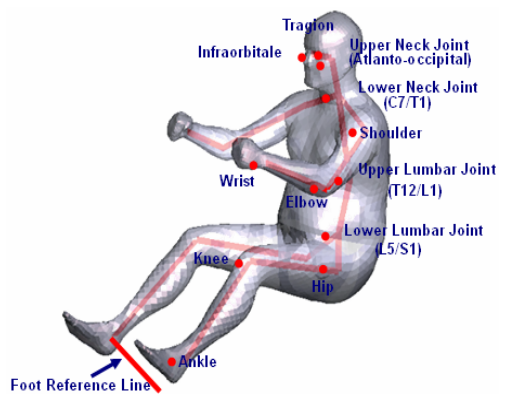

Fig. 7. Kinematic linkage model for $50^{\text {th }}$ percentile male

Comfortable driving postures of $50^{\text {th }}$ and $95^{\text {th }}$ percentile male models were constructed against the package layout of a medium size sedan. As a first step, the eye point and the hip point were calculated from the regression equation in CPM by applying the given package information such as 'Seat Height', 'SW to BOFx' 
(horizontal distance between center of steering wheel and ball of foot), and 'Cushion Angle' together with anthropometry data of $50^{\text {th }}$ percentile male model.

The following experimental coordinate equations proposed by Reed [10] were utilized to determine the torso segmental articulation angles within the fixed eye point and hip point.

$$
\begin{aligned}
& H N(X)=\sum_{i=1}^{4} L_{i} \operatorname{Sin}\left(\theta_{i}+\gamma_{i} \alpha+\beta\right) \\
& H N(Z)=\sum_{i=1}^{4} L_{i} \operatorname{Cos}\left(\theta_{i}+\gamma_{i} \alpha+\beta\right)
\end{aligned}
$$

where $L_{i}$ is the length of each of four segments between hip and head/neck, $\theta_{i}$ is the starting segment orientation from overall segment angles which was obtained by taking the average from the test measurement. $\gamma_{i}$ means the sensitivity on the segment

\begin{tabular}{|c|c|c|c|c|c|c|}
\hline \multirow[b]{2}{*}{ Segments } & \multicolumn{2}{|c|}{ Reed (1998) } & \multirow{2}{*}{$\begin{array}{c}50 \% \text {-ile model } \\
\begin{array}{c}\text { segmental length } \\
(\mathrm{mm})\end{array}\end{array}$} & \multicolumn{3}{|c|}{ Calculated value } \\
\hline & $\begin{array}{c}\text { Theta } \\
\left({ }^{\circ}\right)\end{array}$ & Gamma & & Alpha & Beta & $\begin{array}{c}\text { Segmental angle } \\
\left({ }^{\circ}\right)\end{array}$ \\
\hline Neck & 2 & 0.399 & 128.14 & -1.47 & 1.00 & 2.42 \\
\hline Torso & -3.6 & 0.617 & 297.66 & -1.47 & 1.00 & -3.50 \\
\hline Lumbar & 32.9 & 1.199 & 172.99 & -1.47 & 1.00 & 30.97 \\
\hline Pelvic & 63.4 & 1 & 111.94 & -1.47 & 1.00 & 62.93 \\
\hline
\end{tabular}

Table 5. Articulation angles between eye and hip points of $50^{\text {th }}$ percentile male model

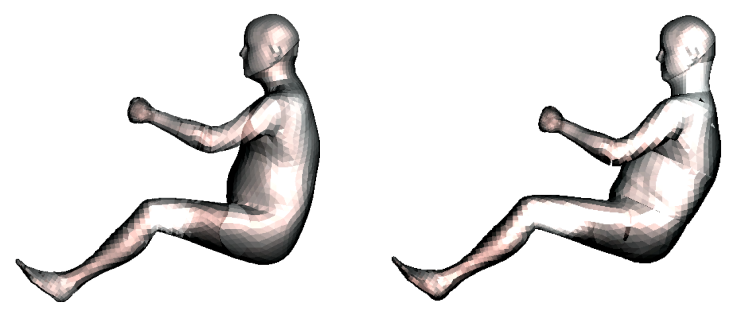

Fig. 8. Scanned and computed comfortable driving postures of $50^{\text {th }}$ percentile male models
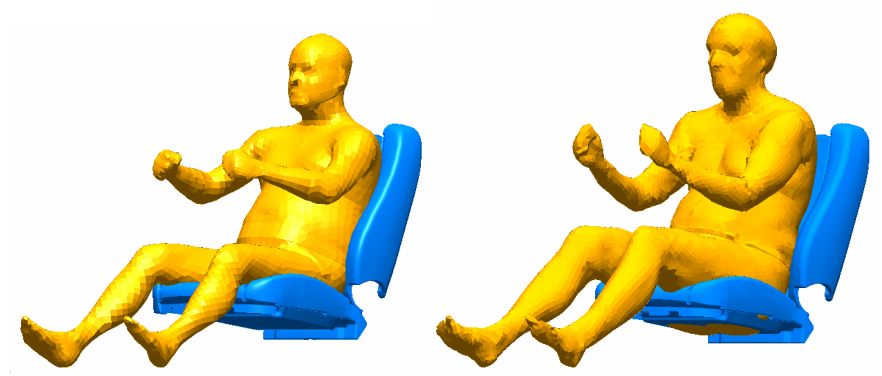

Fig. 9. Computed comfortable driving postures of $50^{\text {th }}$ and $95^{\text {th }}$ percentile male models against a medium size sedan seat 
orientation depending on posture changes and was obtained by dividing each slope value in slope estimate table.

The simulated annealing method was used to compute $\alpha$ and $\beta$ in the above coordinate equations by minimizing the error of eye position relative to the hip point. The optimized segmental angles in the torso region for $50 \%$ tile male model are listed in Table 5.

The process of computing the segmental articulation angles for upper and lower limbs were followed by torso fitting. The pedal angle and restraints of hand position on steering wheel ( 2 and 10 o'clock) were used to decide the posture of legs and arms for a comfortable driving posture. The difference in driving posture of $50^{\text {th }}$ percentile male model between the scanned and the computed are comparatively displayed in Fig. 8. Fig. 9 shows the constructed comfortable postures of $50^{\text {th }}$ and $95^{\text {th }}$ percentile models against a package layout of a medium size sedan.

\section{Finite Element Modeling}

In order to compute the seating kinetics such as body pressure distribution over the seat pad foam, finite element models of $50^{\text {th }}$ and $95^{\text {th }}$ percentile models were developed. A fine mesh with tetragonal solid elements was applied to thigh, buttock and trunk back, the regions in contact with a seat for the body weight support in seating positions and thus the mesh quality of those parts was closely related to the prediction of seating pressure distribution. Skeletons such as femur, pelvis, spine, and rib cage were modeled as rigid bodies and articulated with kinematic joint elements. In particular, the relative placements of femur, pelvic bone, lumbar spine and scapular to skin surface, and visco-elastic deformation of thigh, hip and torso flesh, and the motions of hip joint and lumbar articulation are all taken into account in the modeling process. The basic properties of the lumbar spinal articulation were based of the literature data [11-14]. The mass and inertia properties of body segments were obtained from GEBOD (http://biodyn1.wpafb.af.mil/GebodIntro.aspx) with the user-supplied body dimension option. The other detail FE modeling process of human body for the seating comfort simulation can be found in author's prior publications $[2-4,9]$.

Fig. 10 shows a typical setup for the measurement of body pressure distribution on a raw foam pad (hexahedron in $400 \times 400 \times 100$ (30) mm for the cushion(back)) of the $50^{\text {th }}$ percentile subject in the package layout of a medium size sedan. The subject
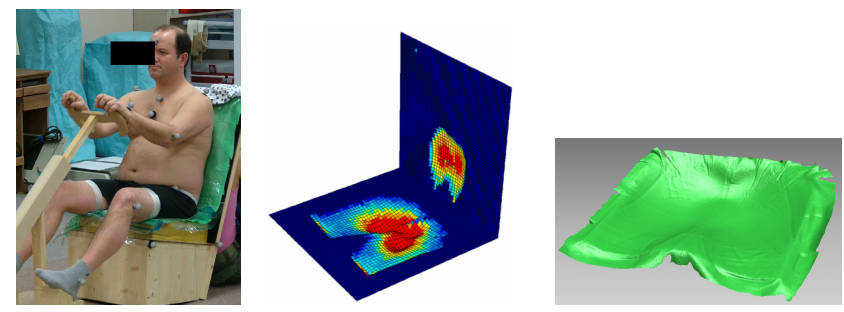

Fig. 10. Test setup for a measurement of body pressure distribution, seating posture, and deformed shape of buttock 

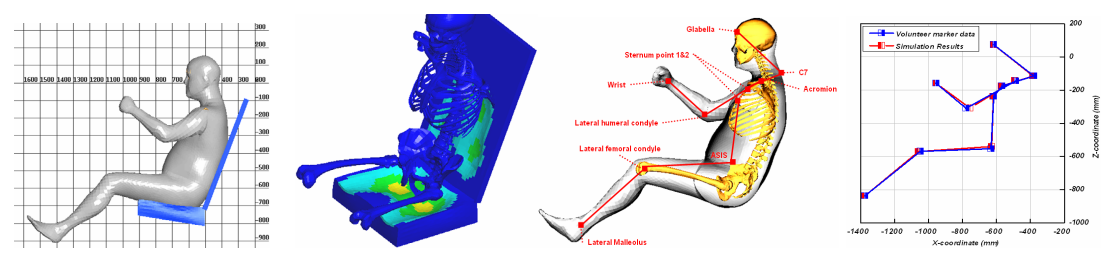

Fig. 11. Simulated seating process with FE human body model

was asked to take a comfortable posture with the given handle and pedal positions. The sitting pressure distributions on both cushion and back were measured by using a pressure sensor system (X-sensor, 43x43 cells). A thin sheet layer of cast (3M's Scotchcast) was also placed between the buttock and raw foam pad for a measurement of deformed buttock shape. The pattern of body pressure distribution in a seating position is generally known to closely depend on the posture of a subject such as torso and thigh angles. Therefore, a precise position of the subjects was measured by an optical sensor system (MotionAnalysis, Model: Eagle 5).

Applying a gravity load into the FE human body model, a simulation of seating process was performed. The foam block in Fig. 11 was modeled through a highly compressible, non-linear elastic material. The material characteristics were determined through preliminary compression tests on a foam sample. A non-linear contact algorithm with friction (Coulomb's law) was used at the interface with the human model. The horizontal (Ux) and vertical (Uz) translations of the wrist and the ankle markers of human body model were fixed to meet the geometric packaging requirement of the vehicle layout. The head was constrained only for the horizontal translation and the pitching (Ry) but free to move in other directions to maintain the natural eye position. The seating posture of the FE human body model in Fig. 11 indicates a good correlation between the experiment and simulation results. A further quantitative analysis of body pressure distribution is now in progress to assess a seating comfort.

\section{References}

1. http://www.tc2.com/what/sizeusa/index.html

2. Montmayeur, N., Haug, E., Marca, C., Choi, H.Y., Sah, S.: Numerical and Experimental Analyses of Seating and Riding Comfort. In: DHMC (2004)

3. Montmayeur, N., Marca, C., Choi, H.Y., and Sah, S.: Experimental and Numerical Analyses of Seating Pressure Distribution Patterns. In: DHMC (2005)

4. Choi, H.Y., Sah, S., Na, S., Montmayeur, N., Marca, C.: Human Body Modeling for Virtual Seat Comfort Testing. In: DHMC (2006)

5. Robbins, D.H.: Anthropometry of Motor Vehicle Occupants, vol. 2: Mid-sized Male, vol. 3: Small Female and Large Male (UMTRI-83-53-2, University of Michigan) (1983)

6. Snyder, R.G., Chaffin, D.B., Schutz, R.: Link system of the human torso. Report no. AMRL-TR-7 1-88. Wright-Patterson Air Force Base, OH: Aerospace Medical Research Laboratory (1972) 
7. Seidl, G.K., Marchinda, D.M., Dijkers, M., Soutas-Little, R.W.: Hip joint center location from palpable bony landmarks-A cadaver study. Journal of Biomechanics 28(8), 995-998 (1995)

8. Bell, A.L., Pedersen, D.R., Brand, R.: A comparison of the accuracy of several hip center location prediction method. Journal of Biomechanics 23(6), 617-621 (1990)

9. Handbook of Numerical Analysis, Computational Models for the Human Body - Human Models for Crash and Impact Simulation, vol. XII, pp. 297-361, Elsevier, Amsterdam (2004)

10. Reed, M.P.: Statistical and biomechanical prediction of automobile driving, $\mathrm{PhD}$ thesis, University of Michigan (1998)

11. Markolf, K.L.: Deformation of the thoracolumbar intervertebral joints in response to external loads. A biomechanical study using autopsy material. J Bone. Jt. Surg. 54-A, 511-533 (1971)

12. Adams, M.A., Dolan, P.: A technique for quantifying the bending moment acting on the lumbar spine in vivo. J. biomechanics 26, 513-522 (1991)

13. Miller, J.A.A., Schultz, A.B., Warwick, D.N., Spencer, D.L.: Mechanical properties for lumbar spine motion segments under large loads. J. Biomechanics 19(1), 79-84 (1986)

14. Panjabi, M.M., et al.: Mechanical Behavior of the Human Lumar and Lumbosacral Spine as shown by Three-Dimensional Load-Displacement Curves. J Bone Jt Surg. 76(A3), 413-424 (1994) 